\title{
L'aménagement du Rhin sur le tronçon entre Bâle et Strasbourg
}

\section{Development of the Rhine between Basle and Strasbourg}

\author{
PAR R. LEFOULON \\ DInECTEUR DE LA RÉGION D'boupEMENT HYDIAULOUE NORD \\ A L'ÉLCCTICITÉ DE FHANCE
}

\begin{abstract}
L'aménagement du Rhin entre Bâle et Strasbourg

L'aménagement du Rhin entre Bàle et Strasbourg, au double titre de la navigation et de l'exploitation de la force hydraulique, est it l'ordre du jour depuis plus d'un siècle.

Sa réalisation n'a pris corps qu'après la premiere guerre mondicle et, entre les deux guerres, seul le premier bief de Kembs a été construit de 1928 a 1932. Depuis 1946, la réalisation du projet se poursuit d'une manière continue et les quatre premiers biefs, représentant $52 \mathrm{~km}$ de canal, sont en service en 1959 . A la cadence actuelle d'exécution, les quatre derniers biefs, qui comporteront chacun une déribation partielle du Rhin, doivent être achevés en 1967. A cette époque, la navigation parcourra sans difficulté, même aux plus hautes eaux, le tronson supérieur entre Bäle et Strasbourg, par le franchissement successif de huit écluses, tandis que les usines hydro-électriques correspondantes produiront en molyenne annuellement plus de 6 milliards $1 / 2$ de $k W h$.
\end{abstract}

Entre Bâle et Strasbourg, le Rhin a une longueur de $125 \mathrm{~km}$ et descend de la cote 244 à la cote 137 , c'est-à-dire présente une chute de $107 \mathrm{~m}$. A partir de Strasbourg et jusqu'à la mer, il ne s'abaisse que de $137 \mathrm{~m}$ sur un cours long de $700 \mathrm{~km}$.

Ces données géographiques expliquent à la fois la violence des inondations sur le troncon Bâle/Strasbourg et les difficultés rencontrées depuis plus de 1000 ans par la navigation pour le parcourir.

Pour remédier à cette situation, des grands travaux d'endiguement du Rhin sont décidés en 1840 par le Roi Louis-Philippe et le GrandDuc de Bade. L'œuvre n'est achevée qu'en 1860; si elle donne une protection efficace contre les
Plans for developing the Rhine between Basle and Strasbourg for both navigational purposes and power production have been under consideration for over a century.

However, no actual work was undertaken until after the first World War, when the first canal section at Kembs was built between 1928 and 1932. Since 1946, work on the scheme has progressed continuously, and the first four conal sections, with a total length of $52 \mathrm{~km}$, are expected to be in service this year. At the present rate of progress, the last four sections -each of which involves partly diverting the river should be ready by 1967 ... Once this is done, no more navigational difficulties will be experienced on the Upper Rhine between Basle and Strasburg, even when the water levels are at their highest. The final layout will include eight successive locks, as well as power stations with a total mean annual output of over 6500 million kWh.

inondations, elle ne résout malheureusement pas le problème de la navigation. Au contraire, elle l'aggrave car le Rhin, resserré entre ses nouvelles digues, accruiert une puissance d"érosion accrue dans les périodes de hautes eaux. L'approfondissement du lit mineur fait apparaître, au bout de quelques années, des fonds rocheux qui entravent la navigation pendant les périodes d'étiage.

La question en est là au début du 20" siècle, au moment où la Suisse aspire à une liaison directe avec la mer et les grands centres métalIurgiques et sidérurgiques de la Ruhr et de la Rhénanie.

A cette même époque, les progrès de la science et de la technique permettent d'envi- 


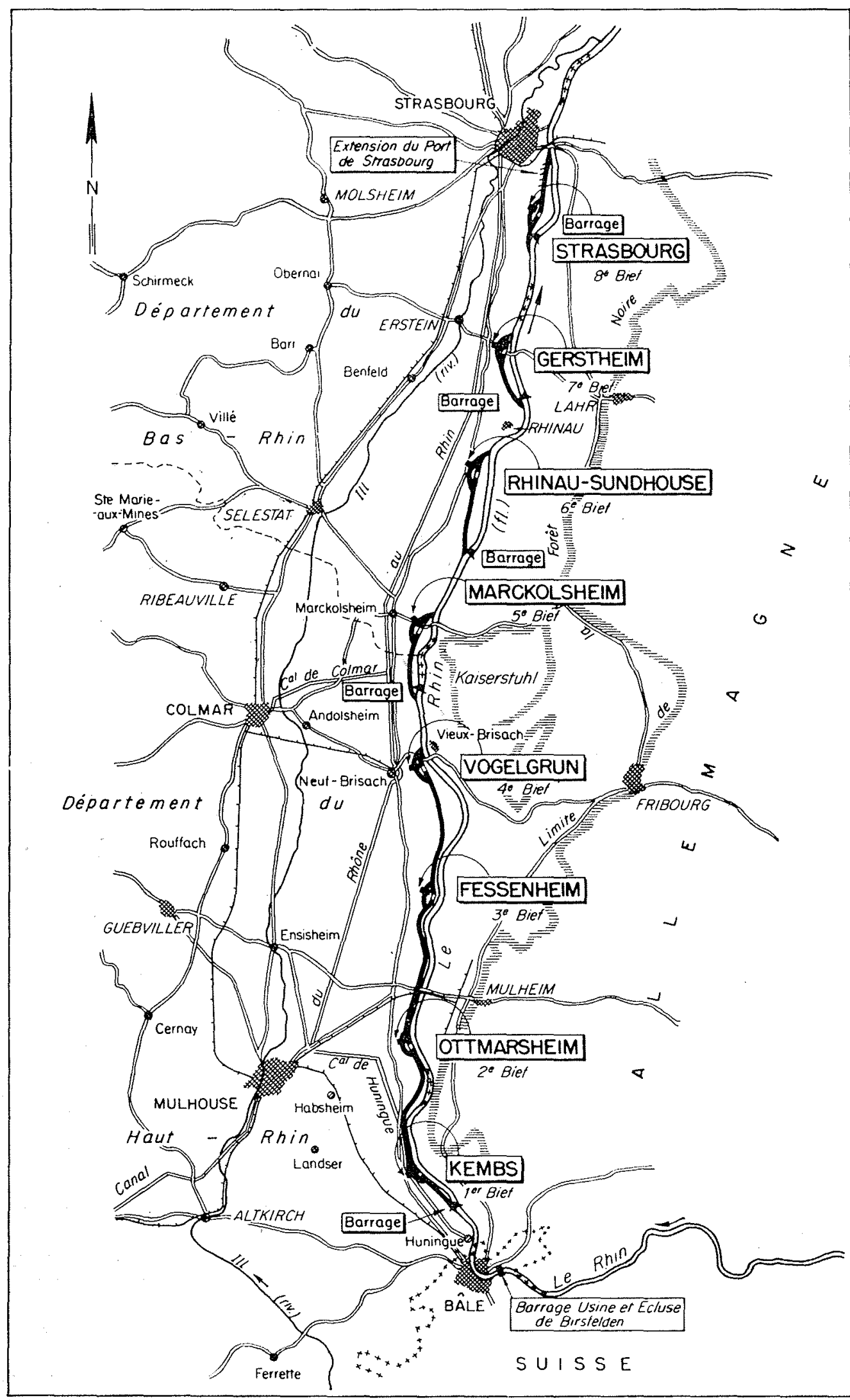

Plan général

l'aménagement. 
En 1953 sont commencés les travaux du $3^{\circ}$ bief de Fessenheim, qui s'achèvent en 1956.

Un nouveau tronçon de $17 \mathrm{~km}$ de canal est alors mis en exploitation avec deux nouvelles écluses semblables à celles d'Ottmarsheim et une usine hydroélectrique produisant également près de 1 milliard de $\mathrm{kWh}$ par an.

Pour ce bief, il a été nécessaire d'exécuter 23 millions de $\mathrm{m}^{3}$ de terrassements et de mettre en place plus de $700000 \mathrm{~m}^{3}$ de béton et $8500 \mathrm{t}$ d'acier.

Dès la fin de 1955 les travaux préparatoires du quatrième bief de Vogelgrun sont entrepris. La mise en service de ce nouvel aménagement est prévue dans le premier trimestre de 1959 ; il créera $14 \mathrm{~km}$ nouveaux de canal. Celui-ci aura alors depuis Kembs une longueur totale de $52 \mathrm{~km}$ constituant, avec sa largeur de $135 \mathrm{~m}$ et sa profondeur de $9,50 \mathrm{~m}$, une des plus importantes réalisations en Europe de ce type d'ouvrage.

Les écluses de Vogelgrün sont analogues à celles des biefs précédents et l'usine hydroélectrique produira annuellement quelque $720 \mathrm{mil}-$ lions de $\mathrm{kWh}$.

La poursuite de l'aménagement du Rhin à l'aval de Vogelgrun ne se fera plus selon le projet de 1925. La France et l'Allemagne ont signé un accord en 1956, aux termes duquel les quatre derniers biefs seront réalisés par dérivation partielle du neuve. Chaque aménagement comportera un barrage et une dérivation sur laquelle seront construits, comme précédemment, des écluses et une usine hydroélectrique. Cette solution donne satisfaction aux deux pays et sera réalisée par la France.

Marckolsheim, cinquième bief, a été entrepris en 1957 suivant ce projet et son exécution se poursuivia jusqu'au cours de 1961.

Les trois derniers biefs doivent être successivement entrepris à la cadence d'un bief tous les deux ans et mis respectivement en service en 1963,1965 et 1967 .

A cette dernière date, l'aménagement aura atteint ses deux grands buts :

$1^{\circ}$ Produire annuellement 6 milliards 700 millions de $\mathrm{kWh}$ avec un effectif de l'ordre de 300 personnes, représentant l'énergie d'une mine de charbon inépuisable, d'une production annuelle de 2 millions $800.000 \mathrm{t}$

2" Permettre presque à toute époque, sur $125 \mathrm{~km}$ de Rhin, une navigation plus facile et plus économique car la traction sur les biefs conduira à économiser les trois quarts du combustible actuellement utilisé à la traction sur le fleuve.

Ces deux buts sont déjà atteints partiellement puisqu'en 1959 la production annuelle d'énergie électrique des quatre premières usines s'élèvera à 3 milliards 400 millions de $\mathrm{kWh}$ et que le tomage des importations par le Rhin du port rhénan de Bâle atteindra un chiffre voisin de 6 millions de tonnes, alors qu'il n'était que de $280.000 \mathrm{t}$ en 1932.

Pour mieux faire connaître l'importance de cette œuvre, les difficultés rencontrées dans sa réalisation, M. Bouchet, ingénieur des Pontset-Chaussées, M. Loubaton, Directeur-adjoint de la Région d'Equipement Hydraulique Nord à l'Electricité de France et M. Lescail, ingénieur en chef à la même Direction, ont traité plus à fond, dans les articles qui suivent, les ouvrages-types de l'ensemble de l'aménagement.

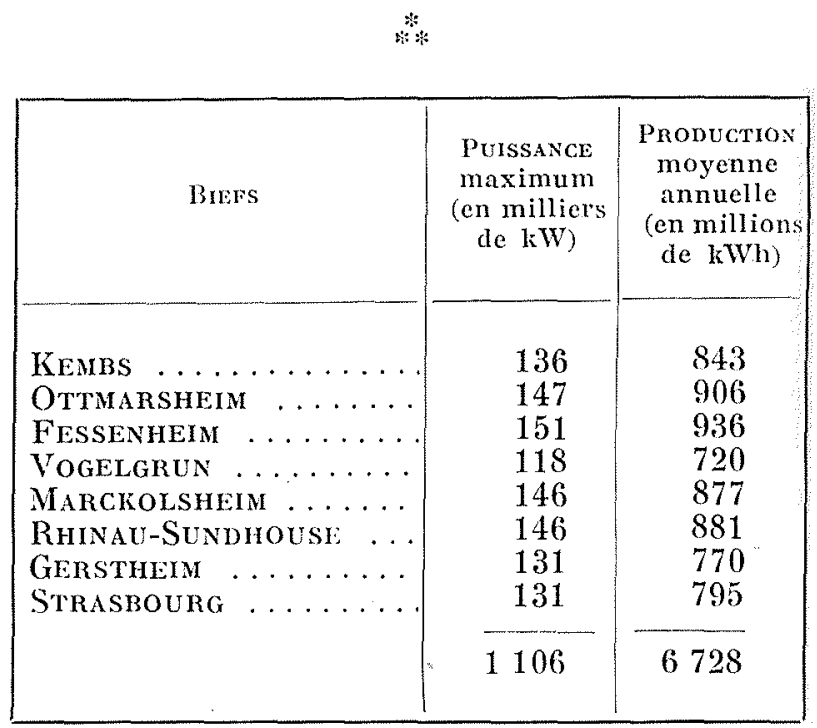

